\title{
III. Parlamentarische Funktionsfelder und die Problemlagen der Inflationszeit
}

Aufbauend auf die strukturgeschichtlichen Skizzen in den vorigen Kapiteln können nun die konkreten Funktionsfelder des Weimarer Reichstags und der französischen Chambre des Députés umrissen werden. Dabei sollen die nationalspezifischen Funktionsweisen beider Parlamente gegenübergestellt, die Problemkonstellationen der Inflationszeit dargelegt und die Fragestellungen für den Zweiten Teil der Untersuchung präzisiert werden.

\section{Regierungstragende Funktion}

Die Ausgestaltung der für den modernen Parlamentarismus so entscheidenden regierungstragenden Funktion zeigt im deutsch-französischen Vergleich erhebliche Abweichungen. Die Teilfunktionen der Bildung, Unterstützung und Abberufung einer Regierung gingen dabei jeweils charakteristische Verbindungen ein, so daß ein isolierter Vergleich nur bedingt aussagekräftig ist und immer der Bezug zum Gesamtkomplex gesucht werden muß. Zu diesem Zweck soll hier idealtypisch von den beiden oben unterschiedenen Grundmustern des klassischen individualisierten und des modernen parteiengestützten Parlamentarismus ausgegangen werden'.

- Beim ersten Typus, der sich bis zum Ersten Weltkrieg in der Dritten Republik durchaus bewährt hatte, steht der häufigen Abberufung der Regierung eine schnelle und flexible Neubildung entgegen. Nach dem Sturz eines Kabinetts erfolgt oft nur eine geringfügige politische und personale Modifizierung der Ministerliste, begleitet von einer entsprechenden Veränderung des Regierungslagers ${ }^{2}$. Der Instabilität der Regierungen entspricht damit eine relative Stabilität des ministeriellen Personals und auch der politischen Grundtendenz ${ }^{3}$. Die Stützung eines installierten Kabinetts durch ein festes Regierungslager hingegen bleibt in der Regel schwach, die losen Regierungsbündnisse - von Koalitionen zu sprechen, wäre hier irreführend - sind nur bedingt deckungsgleich mit Partei- und Fraktionsgrenzen, Ad-hoc-Mehrheiten bilden die Regel. Unter diesen Umständen kommt der alte Dualismus von Parlament und Regierung noch relativ stark zur Geltung. In diesem Parlamentarismus mit fluktuierendem Regierungslager sind die individuellen Abgeordnetenrechte stark ausgeprägt und die politischen Beziehungen in hohem Maße personalisiert, während das Parteiwesen nur in bescheidenem Umfang entwickelt ist.

- Der zweite Typus hingegen ist auf die Existenz gut organisierter Parteien und Fraktionen angewiesen. Die Ausbildung einer stabilen und disziplinierten Regie-

1 Vgl. oben S. 22f. Vgl. auch die Unterscheidung einer „klassisch-liberalen“ und einer „realistischen“ Position der Parlamentarismustheorie in Schütt-Wetschky, Haben wir eine akzeptable Parlamentarismustheorie?

2 Bezeichnend ist der zeitgenössische Begriff der „replâtrage“ (in etwa: Flickwerk).

3 Vgl. Soulier, L'instabilité, S. 480-484; ausführlich hierzu, allerdings mit einem teilweise fragwürdigen positivistisch-statistischen Ansatz: Ollé-Laprune, La stabilité des ministres. 
rungsmehrheit ${ }^{4}$ in Form einer festen Parteienkoalition wirkt als Sicherung gegen den Sturz einer Regierung. Der informelle Konsens zwischen den Koalitionspartnern und eine hohe Fraktionsdisziplin lassen dabei nur noch wenig Spielraum für parlamentarische Deliberation, individualisiertes Verhalten der Abgeordneten oder kleinere Verschiebungen in der politischen Ausrichtung des Regierungslagers. Angesichts einer stabilen Regierungsstützung wird die Regierungsbildung nur noch in großen Intervallen ausgeübt.

Bei genauerer Betrachtung der konkreten Teilfunktionen läßt sich unschwer erkennen, daß die späte Dritte Republik noch eine relativ große Ähnlichkeit zum ersten Muster aufwies, während die Weimarer Republik mit ihrem relativ entwikkelten Parteiensystem bereits eine grundlegende Voraussetzung für das moderne Parlamentarismusmodell erfüllte:

a) Die Regierungsbildung war in Frankreich wie in Deutschland formell eine Angelegenheit des Staatspräsidenten ${ }^{5}$. Weder das deutsche noch das französische Parlament besaßen das Recht, den Regierungschef selbst zu wählen. Die Fraktionen der französischen Abgeordnetenkammer und bis 1930 auch jene der deutschen Nationalversammlung bzw. des Reichstags hatten sich jedoch aufgrund der parlamentarischen Verantwortlichkeit der Regierung weitgehende informelle Mitwirkungsrechte gesichert ${ }^{6}$. Theoretisch war es zwar möglich, daß der Präsident eine Regierung gegen den Mehrheitswillen des Parlaments installierte, die Abgeordneten konnten einem derartigen Kabinett aber sofort das Vertrauen entziehen ${ }^{7}$. In der Praxis des Reichstags und der Abgeordnetenkammer folgte, obwohl dies in der Verfassung jeweils nicht direkt gefordert wurde, auf die erste Präsentation eines neuen Kabinetts eine mehr oder minder explizite parlamentarische Vertrauensbekundung8. Da es in Frankreich kein formelles Mißtrauensvotum gab, besaß diese Praxis hier eine höhere Notwendigkeit als in Deutschland.

In beiden Staaten übernahm der Staatspräsident die Aufgabe eines Managers der Kabinettsbildung, der weitgehend in Einklang mit dem sich abzeichnenden parlamentarischen Regierungslager handelte. Für Frankreich darf die präsidentielle Rolle bei der Auswahl des Regierungschefs nicht unterschätzt werden'. Die geringe Kohäsionskraft der Fraktionen, die Vielfalt politischer Nuancen und die

4 Im Französischen wird hier vom „fait majoritaire“ gesprochen.

5 Art. 53 der Weimarer Reichsverfassung, Art. 3 des Gesetzes vom 25. Februar 1875: „Il nomme à tous les emplois civils et militaires.“ Klarere Vorschriften gab es in den Verfassungsgesetzen von 1871 und 1873. Auch die Krise von 1877 ließ die präsidentielle Ernennung unangetastet.

${ }_{6}^{6}$ Insofern kann gemäß der vorgegebenen Terminologie von einer regierungsbildenden Funktion, nicht aber - wie in der Tradition Bagehots meist üblich - von einer Wahlfunktion des Parlaments gesprochen werden.

7 Vgl. Art. 54 der Reichsverfassung und Art. 6 des Gesetzes vom 25. Februar 1875.

8 In Frankreich geschah dies durch das "Anhängen“ der Vertrauensfrage an eine Gesetzesvorlage oder einen „ordre du jour“; oft handelte es sich nur um einen Geschäftsordnungsantrag. In Deutschland war eine explizite - im Trend aber immer unverbindlicher werdende - Vertrauenserklärung üblich. Beim Antritt der Regierung Marx I wurde ganz darauf verzichtet, die Zustimmung zum vorgelegten Ermächtigungsgesetz galt als Ersatz. Arns, Regierungsbildung, S. 195-204, der auch ausführlich auf die staatsrechtliche Interpretation eingeht, sieht hier einen "fortschreitenden Bedeutungsschwund“. Angesichts der französischen Praxis scheint Arns diese Frage allerdings überzubewerten.

9 Vgl. auch Albertini, Regierung und Parlament, S. 22 f. Neben der schwachen Konturierung des Parteiwesens ist hier auch zu berücksichtigen, daß es traditionell keineswegs üblich war, herausgehobene parlamentarische Persönlichkeiten mit der Funktion des Président $d u$ conseil zu betrauen. 
Bedeutung persönlicher Entscheidungen erforderten geradezu eine koordinierende Instanz und eröffneten relativ große Spielräume bei der Suche nach einem geeigneten Kandidaten. Informelle Beratungen mit den Fraktionen über die Akzeptanz bestimmter Persönlichkeiten blieben aber auch hier unerläßlich, und von einem substantiellen Einfluß des Präsidenten auf die politische Ausrichtung der neuen Regierung kann kaum die Rede sein. Indem der französische Staatspräsident traditionell Vorgespräche mit den Präsidenten von Kammer und Senat führte, war von Anfang an die parlamentarische Mitwirkung bei der Auswahl eines mit der Regierungsbildung zu beauftragenden Politikers gewahrt.

In Deutschland spielten die koalitionspolitischen Vorstellungen des Präsidenten eine nicht unerhebliche Rolle bei der Regierungsbildung ${ }^{10}$. Dennoch ließ die Weimarer Republik trotz mangelnder Erfahrungen mit dem parlamentarischen System die Verfassungsintention eines weitgehend präsidentiell bestimmten Verfahrens erstaunlich schnell hinter sich, was teilweise für heftige Irritationen sorgte und der zeitgenössischen Parlamentarismuskritik immer wieder Auftrieb gab.

Die Kabinettsbildung in der späten Dritten Republik ging meist erheblich leichter vonstatten als in Deutschland ${ }^{11}$. Vor allem zwei Ursachen scheinen hierfür verantwortlich gewesen zu sein: das breitere Spektrum der potentiellen Regierungskräfte und der Umstand, daß die Übernahme eines Ministeramtes in der Regel noch eine individuelle Entscheidung war.

Frankreich befand sich in der günstigen Lage, daß die parlamentarische Republik bereits seit Jahrzehnten fest etabliert war und daß die diversen Monarchismen längst von einer weitgefaßten "republikanischen Synthese"12 aufgesogen oder marginalisiert worden waren. Zur Bildung eines systemloyalen Regierungslagers stand somit im Prinzip fast die gesamte politische Breite der Abgeordnetenkammer zur Verfügung, darunter mit kleinen Einschränkungen auch die rechtsbürgerlichen Kräfte im Umfeld der Fédération républicaine. Diese Grundsituation blieb bis 1940 nahezu unverändert, was auch an den Barrieren des Mehrheitswahlrechts gegen das parlamentarische Erstarken systemfeindlicher Kräfte lag ${ }^{13}$.

Ganz anders gestaltete sich die Situation bekanntlich in der Weimarer Republik. Die parlamentarische Position der systemtragenden Kräfte war hier seit 1920 äußerst fragil. Zwar darf nicht übersehen werden, daß der Weimarer Parlamentarismus durchaus auch integrative Kraft entfaltete und sich so der Kreis der im weiteren Sinn systemloyalen Parteien und damit auch der potentiellen Partner in einer Regierungskoalition sukzessive auf DVP, USPD und zeitweise auch DNVP aus-

10 Deutlich wird dies bereits in Eberts von einem konsenspolitischen Ansatz getragenen Streben nach einer Großen Koalition. Anders war dann die Situation unter Hindenburg. Vgl. allgemein auch die problemorientierte Skizze in Möller, Weimarer Republik, S. 196-199.

11 Ein gewisses äußerliches Indiz hierfür ist der Zeitraum zwischen Abtritt der alten und Antritt der neuen Regierung. Sowohl im Durchschnitt (Deutschland 1919-1930: 15 Tage; Frankreich 1919-1940: 3 Tage) als auch in der Häufung längerer Intervalle lag hier die Weimarer Republik deutlich über der Dritten Republik. Die quälenden deutschen Diskussionen um eine Um- oder Neubildung der Regierung parallel zur Existenz einer amtierenden Regierung sind damit noch gar nicht erfaßt.

12 Vgl. Hoffmann u. a., In Search of France, S. 3-5.

13 Zum Wahlrecht der Zwischenkriegszeit, das 1919 und 1924 starke Momente des Mehrheitswahlrechts enthielt, und dann wieder ganz auf dieses System wechselte, vgl. Huard, Suffrage universel, S. 231-238. 
dehnte. Die Ergebnisse der beiden Reichstagswahlen von 1924 schränkten allerdings die Spielräume der Regierungsbildung erneut empfindlich ein. Schwerwiegend war der Umstand, daß die konservative DNVP primär aus verfassungspolitischen Gründen kein vollwertiger Partner einer bürgerlichen Koalition war. Gerade auch wegen der niemals ganz überbrückten Kluft zur DNVP rückte das sozial- und wirtschaftspolitisch prekäre Modell der Großen Koalition immer wieder in den Mittelpunkt der Diskussion und wurde zum Ziel schwieriger und meist erfolgloser Koalitionsverhandlungen.

Auch hinsichtlich der parlamentarischen Regierungsbereitschaft war die Weimarer Republik dem französischen Nachbarn insgesamt unterlegen. Da es in der späten Dritten Republik immer möglich war, über eine politisch schlankere Konstellation parlamentarische Mehrheiten zu erreichen, lagen auch die Anforderungen an die Kompromißbereitschaft niedriger. Hinzu kam die stärkere personale Prägung der französischen Regierungsbildung: Programmatische Fragen spielten in der Regel meist nur eine geringe Rolle, Parteien und auch Fraktionen hatten traditionell weitaus weniger mitzureden, und die Übernahme eines Ministerpostens war in stärkerem Maße als in Deutschland persönliches Ziel zahlreicher Parlamentarier. Da die Parteiräson im Mitte-rechts-Spektrum kaum ins Gewicht fiel, war die Kabinettsbildung meist ein flexibler Prozeß, der relativ schnell abgeschlossen wurde. Von einer Koalitionsbindung im deutschen Sinne kann dabei allenfalls ansatzweise die Rede sein. Vielmehr handelte es sich traditionell um eine mehr oder minder lose parlamentarische Kooperation zwischen verschiedenen politischen Gruppierungen. Die wichtigsten Konstellationen sind in Tabelle 3 ersichtlich.

Tab. 3: Die wichtigsten parlamentarischen Bündniskonstellationen in der späten Dritten Republik: parteipolitische Spannweiten ${ }^{14}$

\begin{tabular}{|c|c|c|c|c|c|c|}
\hline SFIC & SFIO & PRS & PR & $\mathrm{AD}$ & FR & extreme Rechte \\
\hline \multirow[t]{5}{*}{$\kappa$} & Front & & $\rightarrow 1$ & & & \\
\hline & \multicolumn{6}{|c|}{$\leftarrow$ Cartel des Gauches $\rightarrow \mid$} \\
\hline & & \multicolumn{5}{|c|}{$k$ Concentration $\rightarrow \mid$} \\
\hline & & $\kappa$ & Unio & tionale & $\rightarrow \mid$ & \\
\hline & & $\leftarrow$ & \multicolumn{3}{|c|}{ Bloc national } & $\rightarrow \mid$ \\
\hline
\end{tabular}

Allerdings darf nicht übersehen werden, daß sich in Frankreich gewisse funktionale Veränderungen anbahnten. Das traditionelle Verhalten bei der Regierungsbildung wurde vor allem von der Sozialistischen Partei gesprengt. Zum einen war die Fraktionsdisziplin hier erheblich höher als bei den bürgerlichen Kräften, so daß die Frage einer Regierungsbeteiligung - wie dann später auch bei den Kommunisten - von den Parteigremien und nicht von einzelnen Abgeordneten

14 Dieser grobe Überblick berücksichtigt nicht das komplexe Fraktionswesen der politischen Mitte. Vgl. hierzu Anhang, Tab. 2.2. Der Parti républicain-socialiste (PRS) ging 1935 in der Union socialiste républicaine auf. 
entschieden wurde. Ein Bündnis mit den Sozialisten gewann daher bereits einen koalitionsähnlichen Charakter. Zum anderen tat sich die SFIO nach dem Ausscheiden aus der Union sacrée im Jahre 1917 lange Zeit aus strategischen und teilweise auch ideologischen Gründen sehr schwer mit der direkten Beteiligung an einer „bürgerlichen“ Regierung ${ }^{15}$. So beschränkte sich die Partei in der Kartellzeit 1924/25 auf - durchaus verläßliche - Zusagen für eine parlamentarische Unterstützung gegenüber den vor allem vom Parti radical getragenen Mitte-linksRegierungen sowie auf die Aktivität einer Regierungspartei in den parlamentarischen Ausschüssen.

In Deutschland fiel die Entscheidung zur Regierungsbeteiligung weitgehend durch Partei- und Fraktionsinstanzen; ausschlaggebend war vor allem die Bereitschaft zur Koalitionspolitik. Daß diese im Prinzip parteienstaatlich organisierte Regierungsbildung weniger flexibel war als die immer noch stark personalisierte französische Praxis, liegt auf der Hand. Gerade die Schwierigkeiten bei der Vereinbarung breiter Parteienkoalitionen führten dann aber auch in Deutschland zu Varianten einer stärker personbezogenen Regierungsbildung. Vor allem in den liberalen Parteien, die stark an einem individualisierten Parlamentarismusbild orientiert waren, wurde dies teilweise sehr begrüßt. In derartige Kabinette der „Persönlichkeiten " traten zwar durchaus auch parteipolitisch gebundene Politiker ein, deren Fraktionen begriffen sich jedoch nicht als fester Teil einer Koalition ${ }^{16}$. Im Gegensatz zur französischen Kabinettsbildung alten Typs handelte es sich hier allerdings eher um Notlösungen, denn die parteipolitische Definition des Regierungslagers wurde dadurch kaum verändert. Tabelle 4 gibt einen Überblick über die in der Weimarer Republik auf Reichsebene praktizierten Koalitionstypen.

Tab. 4: Koalitionstypen in der Weimarer Republik auf Reichsebene: parteipolitische Spannweiten

\begin{tabular}{|c|c|c|c|c|c|c|}
\hline \multirow[t]{2}{*}{$\mathrm{KPD}$} & USPD/SPI & DDP & $\mathrm{Z}$ & DVP & DNVP & NSDAP \\
\hline & $\kappa$ & Neimarer Koalition & $\rightarrow 1$ & & & \\
\hline & $\leftarrow$ & Große Ko & & $\rightarrow \mid$ & & \\
\hline & & $\leftarrow$ Bürq & alitio & itte $\rightarrow$ & & \\
\hline & & & $\kappa$ & Rechts & ion $\rightarrow$ & \\
\hline
\end{tabular}

b) Bei der Unterstützung der Regierung durch ein parlamentarisches Regierungslager zeigten sich sowohl in der späten Dritten Republik als auch in der Weimarer Republik nahezu permanent Probleme. Ursachen und funktionaler Stellenwert waren allerdings durchaus unterschiedlich.

In der Dritten Republik besaßen Regierung und Regierungsmehrheit traditionell nur einen geringen Zusammenhalt ${ }^{17}$. Als wesentliche Ursache lassen sich die

$15 \mathrm{Zu}$ Unterschieden in der Bereitschaft zum Kabinettseintritt zwischen SFIO und SPD vgl. Winkler, Demokratie oder Bürgerkrieg, und ders., Klassenkampf versus Koalition.

16 Erstmals erfolgte dieses Vorgehen im Oktober 1921 bei Bildung der Regierung Wirth II. Vgl. dann v.a. die Regierungsbildung Cuno.

$17 \mathrm{Daß}$ hier ein Grundproblem des französischen Parlamentarismus lag, wurde zeitgenössisch und in 
generellen Defizite in der Disziplinierung des Regierungslagers erkennen, was wiederum mit der nur vagen Ausprägung des Parteiwesens, dem weiterhin vorherrschenden dualistischen Verständnis von Parlament und Regierung, dem Paradigma des deliberativen Individualismus sowie der institutionellen Schwäche des Kabinettschefs ${ }^{18}$ zusammenhing. Allzu häufig mußten Regierungen oder einzelne Minister unter diesen Umständen die Vertrauensfrage einsetzen, um für bestimmte Vorhaben die nötige Mehrheit zu erzwingen, was dann schnell und manchmal auch überraschend zum Sturz des Kabinetts führen konnte. Hinzu kam, daß die Regierung nicht nur in der Abgeordnetenkammer, sondern auch im Senat auf Mehrheiten angewiesen war.

Der Weimarer Parlamentarismus war infolge seines relativ disziplinierten Partei- und Fraktionswesens eigentlich für eine stabile Regierungsstützung prädestiniert. Hieraus ergab sich die spezifisch moderne Herausforderung des jungen parlamentarischen Systems, das kaum eine Chance hatte, an die fluktuierenden französischen Verhältnisse anzuknüpfen. Grundsätzlich ist zu bedenken, daß in Deutschland seit den Reichstagswahlen von 1920 die politische Bandbreite von Mehrheitsregierungen stets sehr weit sein mußte, daß aber gleichzeitig die Hindernisse für eine gemeinsame Arbeit des parlamentarischen Regierungslagers - die Verschränkung inhaltlicher und verfassungspolitischer Divergenzen sowie die Häufung sozioökonomischer und außenpolitischer Probleme - äußerst hoch lagen $^{19}$. In der Praxis der Weimarer Regierungsbündnisse konnte die Fraktionsdisziplin nur zeitweise eine stabilisierende Wirkung gewinnen, hatte im verschärften Konfliktfall dann aber einen klaren Bruch zur Folge, entweder durch den geschlossenen Regierungsaustritt einzelner Parteien oder durch den Rücktritt des gesamten Kabinetts. Ein in der Regel geringeres Spannungsverhältnis herrschte innerhalb von Minderheitsregierungen ${ }^{20}$. Allerdings war es immer wieder von neuem notwendig, über die eigentlichen Koalitionsparteien hinaus parlamentarische Mehrheiten zustande zu bringen.

c) Die Abberufung einer Regierung gehörte in der Dritten Republik gleichsam zum parlamentarischen Alltag. Die Dramatik dieses Vorgangs wurde - wie oben skizziert - in der Regel durch die rasche Regierungsneubildung abgemildert. Nach dem Ersten Weltkrieg nahm die Häufigkeit eines Regierungssturzes im Vergleich zu den ersten 50 Jahren der Dritten Republik sogar noch etwas zu. Von 1919 bis 1940 amtierten 45 Regierungen 21, die Mehrzahl von ihnen wurde gestürzt durch Niederlagen bei der Vertrauensfrage in der Abgeordnetenkammer oder auch im Senat ${ }^{22}$. Formelle, von einer der beiden Kammern ausgehende Mißtrauensvoten gab es nicht, sie waren angesichts der allgegenwärtigen Vertrauensfrage

der Literatur nur selten erkannt; eine große Ausnahme ist Soulier, L'instabilité, passim und v.a. S. 578.

$18 \mathrm{Vgl}$. hierzu oben S. 31.

19 Allgemein zu Problemen der Regierungsstützung in der Weimarer Republik vgl. Witt, Kontinuität und Diskontinuität, S. 133-139. Ebd. werden insbesondere auch die latenten Spannungen zwischen Regierung und Regierungsfraktionen betont.

20 In der Politikwissenschaft finden Minderheitsregierungen inzwischen teilweise eine recht positive Bewertung. So betont Strom, Minority Government and Majority Rule, nachdrücklich den Vorzug, Wählerwanderungen zu politischen Extremen abbremsen zu können.

21 Von 1871 bis 1919 waren es 62 Kabinette.

22 Zur gewachsenen Bedeutung des Senats vgl. oben S. 48. 
aber auch nicht nötig. Besonders hoch lag die Frequenz der wechselnden Kabinette in den Jahren 1925-26 (8; Jahresschnitt: 4) und 1931-34 (13; Jahresschnitt: 3,25). Häufig war der Regierungssturz das mehr oder minder überraschende Resultat einer politischen Kräfteverschiebung oder einer Divergenz zwischen der Regierung und ihrer parlamentarischen Basis. Hier von übermäßiger parlamentarischer Macht zu sprechen, erscheint irreführend. Eher handelte es sich um den Ausdruck einer permanenten Funktionsschwäche bei der Kooperation innerhalb des Regierungslagers, wobei nun in der Zwischenkriegszeit mehrfach auch parteipolitische Entscheidungen oder Einflüsse der politischen Öffentlichkeit eine Rolle spielten ${ }^{23}$. Gleichwohl bleibt festzuhalten, daß der Rücktritt eines Kabinetts in der Regel Folge einer parlamentarischen Aktion war.

Die Weimarer Republik war von einem nahezu ähnlich häufigen Wechsel der Regierungen gekennzeichnet - 19 Kabinette in 14 Jahren -, die Ursachen lagen aber meist nicht in einem unmittelbaren parlamentarischen Vertrauensentzug. Als Instrument, um den Rücktritt einer Regierung zu veranlassen, galt nach strenger Auslegung der Verfassung lediglich ein vom Reichstag ausgehender Mißtrauensantrag (Art. 54, Abs. 2). Bis 1930 gab es nur zwei erfolgreiche Mißtrauensvoten, im Mai und Dezember 1926 gegen die Minderheitskabinette Luther und Marx. Die Verweigerung einer - in der Verfassung nicht vorgesehenen - Vertrauensfrage war nach herrschender staatsrechtlicher Auffassung dagegen kein zwingender Grund für eine Demission ${ }^{24}$. Einen solchen Vertrauensentzug gab es in der Geschichte Weimars nur ein einziges Mal. Die Regierung Stresemann trat umstandslos zurück, als das am 23. November von den Regierungsfraktionen beantragte Vertrauensvotum gescheitert war ${ }^{25}$. Insgesamt wurden die Mittel zur parlamentarischen Abberufung einer Regierung in der Weimarer Republik weit weniger genutzt als in der späten Dritten Republik. Vielmehr demissionierten die Regierungen bis 1930 fast immer in Eigeninitiative, meist als Konsequenz auf einen offenkundigen Bruch des Regierungslagers. Angesichts der stärkeren fraktionellen Disziplinierung und der Möglichkeit des parlamentarischen Regierungssturzes wäre es in derartigen Fällen auch kaum aussichtsreich gewesen, die „offene Feldschlacht “26 im Reichstag zu wagen.

Aus den abweichenden Grundmustern der regierungstragenden Funktion, aber auch aus den unterschiedlichen Krisendimensionen ergeben sich für die Analyse im Zweiten Teil differierende Problem- und Fragestellungen. Erst in der Synthese kann daher eine vergleichende Gesamtbewertung der funktionalen Leistungsfä-

23 Vgl. etwa den Rückzug der Radicaux aus der Regierung nach dem Parteitag von Angers 1928. Weitere Beispiele bei Prélot/Boulouis, Institutions politiques, S. $518 \mathrm{f}$. Die ebd. zu findende negative Bewertung („L'éviction du Parlement comme organè gouvernemental“) verkennt, daß es sich hierbei weniger um einen Verfall des parlamentarischen Systems an sich, als vielmehr um Symptome des Wandels vom klassischen deliberativen hin zu einer moderneren Form des Parlamentarismus handelte.

24 Vgl. Anschütz, Verfassung, S. 190.

25 Bei Gusy, Weimarer Reichsverfassung, S. 133, wird dieser Fall irrtümlich dem Mißtrauensvotum nach Art. 54, Absatz 2 zugcordnet.

26 Ein Beispiel für diese zeitgenössisch verbreitete Wendung findet sich in einer Notiz Erich KochWesers (DDP) vom 16.5. 1924 rückblickend zum Sturz Stresemanns 1923; BA Koblenz, Nl. Koch-Weser, Nr. 30, B1. 19. 
higkeit erfolgen. Ein direkter Vergleich einzelner parlamentarischer Vorgänge, nochmals sei es betont, würde schnell zu analytischen Kurzschlüssen führen.

Auf der deutschen Seite, wo mit einem weit entwickelten Parteiwesen bereits wesentliche Voraussetzungen für das parteiengestützte Parlamentarismusmodell erfüllt waren, werden vor allem Fragen der Koalitionspolitik sowie die damit verbundenen Probleme bei der Bildung und Stützung von Regierungen im Mittelpunkt stehen. In diesem Zusammenhang ist auch die in der Literatur dominierende These von der mangelhaften Kompromißfähigkeit der deutschen Parteien zu prüfen. Umgekehrt ist den Fragen nachzugehen, ob das in der bürgerlichen Mitte vorherrschende Paradigma einer weitgespannten bürgerlich-sozialdemokratischen Regierungskooperation nicht zu einer Überforderung bei der Koalitionsbildung geführt und inwieweit es hierzu Alternativen gegeben hat.

Für die französische Seite stellt sich einerseits die Frage, inwieweit das regierungstragende Funktionsgefüge des klassischen Parlamentarismus von einem allmählichen Veränderungs- bzw. Modernisierungsprozeß erfaßt worden ist. Andererseits ist aber auch auf die fortbestehende Traditionslinie fluktuierender Mehrheitsverhältnisse und flexibler Regierungsumbildungen zu achten. Entscheidende Bedeutung kommt hier immer wieder dem parlamentarischen Stimmverhalten zu, insbesondere in den zahlreichen Vertrauensvoten. Detaillierte Betrachtung verdient auch die jeweilige Zusammensetzung der Kabinette ${ }^{27}$, spiegelte sie doch meist auf subtile Weise jede Verschiebung des politischen Klimas in der Abgeordnetenkammer.

\section{Alternativfunktion}

Auch diese parlamentarische Aufgabe kann in der Realität sehr unterschiedlich wahrgenommen werden. Die beiden skizzierten Grundtypen bei der Ausgestaltung der regierungstragenden Funktion 28 lassen sich mit komplementären Varianten der Alternativfunktion verbinden ${ }^{29}$. Im Falle des individualisierten parlamentarischen Systems alten Typs formt sich die Opposition nur von Fall zu Fall und bietet so inhaltlich gewissermaßen "Ad-hoc-Alternativen“. Politisch oft nur in Nuancen meßbare Alternativen der Regierungszusammensetzung ergeben sich dabei gleichsam aus dem Stand durch die häufige Um- und Neubildung der Kabinette. Der moderne parteienstaatliche Parlamentarismus tendiert hingegen zu einer verfestigten und disziplinierten Opposition, die dem Regierungslager blockartig entgegensteht, inhaltliche Alternativkonzepte entwickelt und systematisch einen Regierungswechsel anstrebt ${ }^{30}$.

27 Grundlage sind die Listen in Bonnefous, Histoire 3, S. 439-445, und ders., Histoire 4, S. 386-392, die einzige vollständige Aufstellung in der Literatur. Die hier zahlreich zu findenden Fehler in der Fraktionsbenennung (besonders häufig: Gauche radicale statt Gauche démocratique) wurden korrigiert, ohne daß dies im folgenden im einzelnen vermerkt wird.

28 Vgl. oben S. $101 \mathrm{f}$.

29 Vgl. auch Steffani, Parlamentarische und präsidentielle Demokratie, S. 241, zu unterschiedlichen Oppositionsformen.

30 Vgl. ebd., S. 242. Die Aussage Steffanis, das parlamentarische System „verlange“ - im Gegensatz zum Präsidialsystem - nach systematischer Opposition, ist für die Gegenwart im Prinzip sicher zutreffend, verkennt aber die Qualitäten einer traditionellen Ad-hoc-Opposition in einem fluktu- 
Erneut ist festzustellen, daß eine klare Zuordnung des französischen und des deutschen Parlamentarismus der Zwischenkriegszeit zu einem der beiden Typen kaum möglich ist. Im Prinzip zeigt sich aber ein ähnliches Bild wie im Falle der regierungstragenden Funktion. Der Parlamentarismus der späten Dritten Republik befand sich im langsamen Übergang vom Modell der vielen kleinen Ad-hocAlternativen hin zu einer systematischeren Erfüllung der Alternativfunktion durch große Oppositionsblöcke. Grundsätzlich erleichtert wurde dieser Prozeß dadurch, daß dank des ausgedehnten republikanischen Spektrums und dank der auch im Wahlrecht begründeten parlamentarischen Schwäche der politischen Extreme stets eine starke loyale Opposition existierte. Selbst in der Periode der breiten Bloc-national-Kabinette stand mit den Sozialisten immer eine große systemloyale Kraft in der Opposition; gerade in der schwierigen Phase nach der kommunistischen Abspaltung von 1920 konnte sich die SFIO damit parlamentarisch als alternative Kraft in Szene setzen.

Der Weimarer Parlamentarismus wäre angesichts des ausgebildeten Parteiwesens ein geeignetes Feld für den modernen Typus einer systematischen Opposition gewesen. Infolge der zunehmenden Schwäche der loyalen Kräfte und deren Absorption durch die Regierungsverantwortung gab es allerdings nur wenige Zeiträume, in denen sich eine eindeutig systemloyale Partei in klarer Oppositionsstellung befand ${ }^{31}$. Insgesamt wurde die Opposition zur Regierungspolitik - parlamentarisch und immer mehr auch außerparlamentarisch - weitgehend eine Angelegenheit der nur unzureichend in das parlamentarische System eingebundenen DNVP und später dann vor allem der KPD und NSDAP32.

Auch im Bereich der Alternativfunktion wird für die französische Abgeordnetenkammer in besonderer Weise darauf zu achten sein, inwieweit sich eine allmähliche Modernisierung der Funktionsweise angebahnt hat. Dabei stellt sich einerseits die Frage nach der Kohärenz parlamentarischer Opposition, andererseits jene nach der Beharrungskraft traditioneller Verhaltensmuster einer „Ad-hocOpposition“. Für den deutschen Reichstag ist vor allem eine Bestandsaufnahme vorzunehmen: In welchem Umfang und in welchen Formen konnte sich in der Inflationszeit überhaupt eine systemloyale Opposition bilden? Gab es nicht vielleicht doch Spielräume und konkrete Optionen für eine stärkere parlamentarische Formierung loyaler Opposition?

ierenden Mehrparteiensystem wie der Dritten Französischen Republik. - Auch bei einer systematischen Opposition werden sich kooperative und kompetitive Strategien immer überlagern.

31 Zur Problematik vgl. Kirchheimer, Germany: The Vanishing Opposition, S. 238-240; Besson, Regierung und Opposition in der deutschen Politik; zur Phase 1924-28 auch Stürmer, Koalition und Opposition.

32 Wichtigste Ausnahme waren die Jahre 1925 und 1927-28, als SPD und DDP jeweils gegen eine bürgerliche Rechtskoalition standen. In Phasen einer bürgerlichen Minderheitskoalition der Mitte geriet die SPD dagegen mehrfach in eine Rolle zwischen Regierung und Opposition, was eine inhaltliche Erfüllung der Alternativfunktion erschwerte. Vgl. Stürmer, Koalition und Opposition. Dennoch kann das relativ gute Wahlergebnis der SPD 1928 sicherlich auch durch die verbesserte Profilierung seit 1924 erklärt werden. 


\section{Legislative Funktion}

Nach den Verfassungen der Weimarer Republik und der Dritten Französischen Republik war die Legislative grundsätzlich Aufgabe des Parlaments ${ }^{33}$. Besonders konsequent war dabei die Dritte Republik, die das Prinzip der im Parlament verkörperten volonté générale nahezu vollständig umsetzte. Der präsidentielle Einfluß auf die Gesetzgebung beschränkte sich im wesentlichen auf das Initiativrecht und die Möglichkeit, eine erneute legislative Behandlung durch das Parlament zu verlangen ${ }^{34}$. Wie bereits erwähnt, blieben plebiszitäre Mitwirkungsmöglichkeiten völlig ausgeschlossen. In der Weimarer Verfassung waren hingegen ein weitergehendes - freilich nie praktiziertes - Vetorecht des Präsidenten ${ }^{35}$ sowie ein in der Realisierung nicht über die Stufe des Volksbegehrens hinausgekommenes Potential der „Volksgesetzgebung“ vorgesehen ${ }^{36}$. Während verfassungsrechtlich das Parlament in beiden Staaten gleichsam den Gipfel seiner Gesetzgebungsfunktion erreicht hatte, führte die moderne Gewaltenverschränkung zwischen Regierung und Parlamentsmehrheit, die in England bereits Ende des 19. Jahrhunderts eine "fast omnipotente Kontrolle des Kabinetts über die Gesetzgebung" bewirkt hatte ${ }^{37}$, tendenziell wieder zu einem steigenden Einfluß der Regierung.

Ein erheblicher Unterschied zwischen den nationalen Gesetzgebungsverfahren lag darin, daß die Dritte Republik den komplizierten Regeln eines Zweikammersystems zu folgen hatte, während in der Weimarer Republik der Reichstag die entscheidende parlamentarische Instanz bildete und das schwach gestaltete Einspruchsrecht des Reichsrats allenfalls aufschiebende Wirkung hatte. In Frankreich besaßen Abgeordnetenkammer und Senat das Recht der Gesetzesinitiative, und von beiden Kammern mußten gleichlautende Gesetzesvorlagen gebilligt werden, die zuvor als "navette“ $z$ wischen den parlamentarischen Lesungen beider Organe zirkuliert waren. Auch wenn die Abgeordnetenkammer de facto ein klares legislatives Übergewicht besaß, waren beide Kammern formal nahezu gleichberechtigt ${ }^{38}$. Daß ein derartiges Verfahren den Prozeß der parlamentarischen Deliberation oft erheblich in die Länge zog oder sogar gänzlich blockierte, ist offensichtlich ${ }^{39}$.

33 Zur Dritten Republik fehlt bislang eine hinreichende verfassungsgeschichtliche Skizze der ordentlichen Gesetzgebung. Vgl. daher die Darstellungen in zeitgenössischen Staatsrechtslehren, $z$. B. in Barthélemy/Duez, Traité élémentaire, S. 558-576. Zur Weimarer Republik va. die Überblicksskizze von Mußgnug, Beziehungen zwischen Parlament, Regierung und Verwaltung, S. 316-321; Gusy, Weimarer Reichsverfassung, S. 144-163; problematisch, weil stark im Sinne der vom Konstitutionalismus geprägten Kritik am ausgedehnten parlamentarischen Gesetzgebungsrecht: Huber, Verfassungsgeschichte 6, S. 403-450.

34 Vgl. Art. 3 des Gesetzes vom 25. 2. 1875 und Art. 7 des Gesetzes vom 16. 7. 1875.

35 Das in Art. 73 WRV vorgesehene präsidentielle Verlangen nach einem Volksentscheid besaß das Potential eines absoluten Vetos.

36 Vgl. oben S. $52 \mathrm{f}$.

37 Blake, The Development of the British Parliamentary System 1861-1901, S. 116.

38 Dies resultierte v.a. aus dem Umstand, daß die Regierungsbildungen weitestgehend von den dort gegebenen Mehrheiten abhängig waren. Obwohl die Regierung als Ausdruck formaler Gewaltenteilung selbst kein Initiativrecht hatte, war sie auch in Frankreich eine Triebkraft der Gesetzgebung, die ihren Anfang in der Regel über eine regierungsnahe Initiative in der Abgeordnetenkammer nahm. Anders die Konstruktion in Art. 68, Abs. 1 WRV: „Die Gesetzesvorlagen werden von der Reichsregierung oder aus der Mitte des Reichstags eingebracht."

39 Vgl. Zweiter Teil, B, Kap. II.3 zur Situation 1924/25. 
Konsequenzen ergaben sich für den Gesetzgebungsprozeß auch aus der jeweiligen Kohärenz der Regierungslager. Insgesamt war die legislative Beratung im französischen Parlamentarismus ein schwer zu kalkulierender Vorgang. Dies betraf nicht allein die Unsicherheiten im Abstimmungsverhalten. Abänderungsanträge („amendements“) von Regierungsvorlagen, die den Gesetzgebungsprozeß verzögerten, gingen oft auch von Abgeordneten des Regierungslagers aus. Differenzen sind schließlich auch hinsichtlich der Ausschußberatung von Gesetzentwürfen festzustellen. In der Dritten Republik, wo das Kommissionswesen eine traditionelle Stütze des deliberativen Parlamentarismus darstellte ${ }^{40}$, war diese in der Regel obligatorisch. Nicht selten kam es dabei zu überraschenden Entwicklungen. Der stets auf drei Plenarlesungen angelegte Weimarer Gesetzgebungsprozeß sah hingegen in den meisten Fällen nur eine fakultative Ausschußbehandlung vor ${ }^{41}$.

Die Abgabe legislativer Kompetenzen an die Regierung und die daraus folgende "vereinfachte Gesetzgebung" auf dem Verordnungsweg bildeten in fast allen parlamentarischen Demokratien Europas eine charakteristische Erscheinung der Zwischenkriegszeit ${ }^{42}$. Besonders ausgeprägt waren diese Vorgänge bekanntlich in der Weimarer Republik, wo schon sehr früh - in den Krisenjahren 1922-24 - eine zeitweise Entparlamentarisierung der Gesetzgebung stattfand. Ab 1930 ist dem Reichstag die legislative Funktion dann nahezu völlig entzogen worden. Auch in Frankreich liegt der "Einstieg“ in die Praxis der décrets-lois, die erst ab 1934 zeitweise ausuferte, bereits in der Krisenphase der Inflationszeit. Wie auch in anderen Staaten gibt es sowohl in der Weimarer als auch in der Dritten Republik einen Zusammenhang mit den drängenden Anforderungen der Währungsstabilisierung. Der dabei zu beobachtende Funktionsverzicht des Reichstags und der Abgeordnetenkammer berührte die klassische Aufgabe des Parlaments und wurde zeitgenössisch mit großer Aufmerksamkeit registriert ${ }^{43}$.

Die Bewertung fällt heute insofern nicht immer leicht, als die Verlagerung legislativer Befugnisse auf die Regierung bis zu einem gewissen Grade die Normalität des modernen Parlamentarismus spiegelt. Man macht es sich daher zu einfach, wenn man hier für die Weimarer Republik und die späte Dritte Republik lediglich einen Krisenprozeß sieht, der auf die großen Ermächtigungsgesetze für die Regierungen Hitler und Pétain zulief. Eine „finalistische" Perspektive wäre hier sicher verfehlt. Kein Zweifel kann allerdings darüber bestehen, daß die Entwicklung schon vor den parlamentarischen Kapitulationsakten von 1933 und 1940 eine höchst problematische Wendung genommen hatte.

40 Grundlegend immer noch die zeitgenössische Darstellung in Barthélemy, Essai sur le travail parlementaire.

41 Vgl. Jekewitz, Ein ritualisierter historischer Irrtum. - Für Frankreich drängt sich der Eindruck auf, als würden die Defizite in der parteipolitischen Durchdringung des parlamentarischen Systems durch eine Hyperaktivität der Ausschüsse aufgefangen.

42 Zu dem Europa und auch die USA betreffenden Phänomen vgl. allgemein Tingstén, Les pleins pouvoirs.

43 Bezeichnend ist die Fülle an zeitgenössischer staatsrechtlicher Literatur. Vgl. für die deutsche Seite besonders die Berichte von Poetzsch (ab 2: Poetzsch-Heffter), Staatsleben 1, S. 206-216; 2, S. 75-77; 3, S. 127-142. Zu Frankreich vgl. Anm. 44. 
Grundsätzlich sind zwei Hauptformen zu unterscheiden, in denen die legislative Funktionsabgabe erfolgt ist: parlamentarische Ermächtigungen an die Regierung zum Erlaß gesetzvertretender Rechtsverordnungen (décrets-lois) sowie die Duldung eines aus der Verfassung abgeleiteten Notverordnungsrechts der Regierung. Für ersteres bestand weder in der Weimarer Reichsverfassung noch in den Verfassungsgesetzen der Dritten Republik eine feste Grundlage, vielmehr entwikkelten sich in beiden Staaten entsprechende Verfahren in einer verfassungsrechtlichen Grauzone ${ }^{44}$. In der Weimarer Republik, wo es vor allem das bereits erwähnte Vorbild der großen Kriegsermächtigung vom 4. August 1914 gab, waren in den Jahren 1919-1921 zunächst einige spezielle Ermächtigungen zur Regelung der Abtretung Elsaß-Lothringens, zur Durchführung der Waffenstillstandsbedingungen und zu Fragen der Übergangswirtschaft verabschiedet worden ${ }^{45}$. Der Durchbruch zu weitgefaßten legislativen Vollmachten erfolgte freilich, wie später genau zu verfolgen sein wird, erst im Jahr 1923. In der Dritten Republik hatte es im Zeichen eines ausgeprägten Gewaltenteilungsdenkens jahrzehntelang eine strikte Ächtung parlamentarischer Ermächtigungen gegeben ${ }^{46}$. Erste kleine Aufweichungen fanden in Form sehr eng umgrenzter Vollmachten während des Ersten Weltkriegs sowie 1919 im Zuge der Eingliederung Elsaß-Lothringens statt ${ }^{47}$. Im politischen Bewußtsein waren diese Fälle aber kaum präsent, so daß erst 1924 der eigentliche Prozeß der Enttabuisierung begann.

Auch ein Notverordnungsrecht gab es streng genommen in keiner der beiden Verfassungen. Allerdings boten in der Weimarer Republik die ursprünglich für den militärischen und polizeilichen Ausnahmezustand gedachten präsidentiellen Befugnisse des Artikels 48 bekanntlich ein Einfallstor für ein bewußt nicht in die Verfassung aufgenommenes Notverordnungsrecht der Regierung ${ }^{48}$. Wiederum lag der Beginn dieser Praxis und der damit verbundenen Stärkung des Reichspräsidenten in der Inflationszeit. Die Verfassungsgesetze der Dritten Republik enthielten keinen Ansatzpunkt für ein eigenständiges Notverordnungsregime der Exekutive, so daß hier die legislative Funktionsabgabe auf den Weg der Ermächtigung beschränkt blieb.

Eine wesentliche Aufgabe der folgenden Kapitel wird es sein, den Prozeß der legislativen Funktionsabgabe während der Inflationszeit detailliert zu analysieren.

$44 \mathrm{Vgl}$ allgemein zu den deutschen Ermächtigungen va. Huber, Verfassungsgeschichte 6, S. 437-443; Frehse, Ermächtigungsgesetzgebung (ebd., S. 185, Überblickstabelle zu allen Weimarer Ermächtigungsgesetzen) sowie Mößle, Verordnungsermächtigung. - Zu den französischen Ermächtigungen vgl. v.a. Mignon, La pratique des décrets-lois; Rusu, Décrets-lois (ebd. passim auch Abdruck der Gesetzestexte); die ältere Literatur zusammenfassend: Rothenfluh, Notrecht, S. 70-79. Kurzer Überblick auch in Soubeyrol, Les décrets-lois sous la Quatrième République, S. 24-27, und in Gicquel, Droit constitutionnel, S. $476 \mathrm{f}$. Zu den Ermächtigungen der dreißiger Jahre vgl. auch unten S. 562-564.

45 Vgl. Frehse, Ermächtigungsgesetzgebung, S. 47-79.

46 Das Gesetz vom 25. 2. 1875 legt in Art. 1 lakonisch fest: „Le pouvoir législatif s'exerce par deux assemblées: la Chambre des députés et le Sénat." Die französische Staatsrechtslehre sah hier in den crsten Jahrzehnten der Dritten Republik keinerlei Spielraum für parlamentarische Ermächtigungen.

47 Vgl. die in Anm. 44 genannte Literatur. Bock, Parlementarisme de guerre, geht auf diese Vollmachten nicht ein.

$48 \mathrm{Zu}$ Notverordnungen werden hier, wie meist üblich, auch Verordnungen im Sinne der „Diktaturgewalt" nach Art. 48 gerechnet. Zur Unterscheidung vgl. Huber, Verfassungsgeschichte 6, S. $435 \mathrm{f}$. 
In welchen Formen und in welchem Umfang, so ist zu fragen, wurde in dieser Phase eine dem Parlament entzogene Rechtsetzung praktiziert? Worin lagen die Ursachen und Motive hierfür, inwieweit mußten mentale Widerstände überwunden werden, und in welcher Weise erfolgte die parlamentarische Durchsetzung von Ermächtigungsgesetzen?

\section{Kontrollfunktion}

Die Kontrolle der Regierung bildet einen eng mit den anderen parlamentarischen Funktionen verzahnten Aufgabenbereich, besitzt aber auch ein spezifisches Instrumentarium, vor allem in Form von Interpellationen und Untersuchungsausschüssen. Allgemein kann festgestellt werden, daß die parlamentarischen Kontrollmöglichkeiten in beiden Staaten weit entwickelt waren. Das zeitgenössisch trotz der Parlamentarisierung der Regierungsverantwortung noch stark ausgeprägte dualistische Verständnis von Parlament und Regierung fand so seine verfassungsrechtliche Entsprechung. Allerdings ist zu bedenken, daß - ähnlich wie bei der Gesetzgebung - ein Formwandel der Kontrollfunktion gleichsam in der Logik der modernen Entwicklung lag. Infolge der engeren Verbindung von Regierung und Regierungsmehrheit ist im Prinzip von einer gewissen Abschwächung und Differenzierung der parlamentarischen Kontrolle auszugehen, die nun, insbesondere in Form der Kritik an der Regierung, idealtypisch überwiegend von der loyalen Opposition ausgeübt wird. In der Realität freilich war auch innerhalb des Regierungslagers noch ein relativ starkes Bewußtsein einer parlamentarischen Gesamtverantwortung für die Kontrollfunktion festzustellen.

Dies gilt besonders für die späte Dritte Republik. Da hier weiterhin Verhaltensmuster einer traditionellen Ad-hoc-Opposition üblich waren, wurde die Kontrolle in gewissem Maße noch in alter Form durch das Parlament als Ganzes wahrgenommen. Vor allem Interpellationen und schriftliche Anfragen als wichtige Instrumente des individualisierten Parlamentarismus besaßen noch hohen Stellenwert und konnten auch von einzelnen Abgeordneten vorgebracht werden ${ }^{49}$. Nicht selten entwickelten sich dann im Rahmen von Interpellationen, die für die Regierungen immer einen Unsicherheitsfaktor darstellten, intensive Debatten zu zentralen politischen Themen. Bedeutsam für die Ausübung der Kontrollfunktion war auch das ausgeprägte Ausschußwesen der späten Dritten Republik, insbesondere wenn es zur Anhörung („audition“) von Regierungsmitgliedern in Ausschußsitzungen kam $^{50}$.

Im Weimarer Reichstag waren die kaiserzeitlichen Möglichkeiten der Kontrolle vor allem im Bereich des Untersuchungsrechtes erweitert worden ${ }^{51}$. Insgesamt war das Kontrollinstrumentarium aber etwas schwächer entwickelt als in Frank-

49 Duguit, Traité, S. 378-390. Zum langfristigen Bedeutungsgewinn schriftlicher Anfragen - als Indiz für die schwindende Deliberation - vgl. Henry, Les questions écrites des députés.

so Vgl. z. B. die über 250 maschinenschriftliche Protokollseiten umfassende „audition“ von Briand und Caillaux zum geplanten und schließlich gescheiterten Ermächtigungsgesetz im Juli 1926; AAN Paris, Procès-verbaux de la Commission des Finances, XIIIe Législature (1926), 16. 7. 1926. Einen Überblick über die Ausschußtätigkeit ab 1926 bietet das Bulletin des Commissions, Paris $1926 \mathrm{ff}$. (zugänglich in AAN Paris).

51 Vgl. Mußgnug, Beziehungen, S. 321-325. 
reich. So gab es, wie bereits erwähnt, gemäß der traditionellen Sonderstellung der militärischen Gewalt keinen parlamentarischen Ausschuß mit spezieller Zuständigkeit für das Militärwesen. Auch von der grundsätzlich geringeren Bedeutung des Weimarer Kommissionswesens war schon die Rede. Das neuartige Mittel der Untersuchungsausschüsse blieb alles in allem wenig effektiv und stieß in der politischen Öffentlichkeit von Anfang an auf starke Vorbehalte, zumal im deutschen Staatsrecht gegen die vermeintliche Beeinträchtigung der richterlichen Gewalt Front gemacht wurde ${ }^{52}$. Interpellationen besaßen bei weitem nicht den Stellenwert wie in der Dritten Republik, was nicht zuletzt an der geforderten Mindestzahl von 30 Unterzeichnern ${ }^{53}$ und an der stärkeren fraktionellen Disziplinierung lag.

Im folgenden wird vor allem darauf zu achten sein, inwieweit es in der Krisensituation der Inflationszeit zu einer Einschränkung parlamentarischer Kontrollmechanismen gekommen ist.

52 Überblick über die eingesetzten Ausschüsse in Poetzsch (ab 2: Poetzsch-Heffter), Staatsleben 1, S. 121-123, 2, S. 75-77. Allgemein zum Instrument vgl. Steffani, Die Untersuchungsausschüsse des Preußischen Landtages, v.a. S. 13-18, 76-80. Steffanis Hauptthese vom agitatorischen Mißbrauch der Untersuchungsausschüsse durch die radikalen Parteien gegen Ende der Republik bedürfte für die Reichsebene noch immer der Verifizierung. Zur zeitgenössischen Kritik vgl. v.a. Schröder, Das parlamentarische Untersuchungsrecht der Weimarer Reichsverfassung.

53 Vgl. Geschäftsordnung des Reichstags vom 12.12. 1922, \55. 